\title{
Innovative Teaching Reform of Embedded System Course -Taking Robot Project as Teaching Template
}

\author{
Zeng Xiangjin*, Cai Dunbo, Lu Tao \\ School of computer science and engineering, Wuhan Institute of Technology \\ * Corresponding author: xjzeng21@163.com
}

Keywords: Embedded; Teaching reform; Project design; Robot platform

\begin{abstract}
According to the characteristics and requirements of the embedded industry, this paper explores the innovative teaching mode of embedded system course, which improves the teaching quality and students' interest in learning by designing the teaching content and adopting the project of robot platform.
\end{abstract}

\section{Introduction}

At present, many universities offer courses related to embedded systems. However, most schools still cultivate students with traditional teaching modes and teaching contents, which makes them unable to learn the basic knowledge and practical skills well, resulting in poor practical ability and weak scientific research and innovation ability ${ }^{[1]}$. Quite a few students even do not recognize school education and choose skills training institutions. Therefore, it is urgent to reform the corresponding teaching content, teaching mode and innovative teaching means of embedded system course ${ }^{[2]}$.

\section{Present Analysis}

Combining the teaching and curriculum analysis in recent years, the disadvantages of traditional embedded system courses are:

(1) The curriculum is unreasonable, many early basic courses have not opened or the content of the narrative is too small, began to learn more demanding professional courses ${ }^{[3]}$. For example, for embedded system courses, students need to learn circuit theory, analog electronic technology, digital electronic technology, microcontroller, $\mathrm{C}$ or $\mathrm{C}++$ in advance; however, many students reflect that the abovementioned courses teachers have less lecture content, experimental foundation is not well laid, which directly leads to the following embedded system courses are somewhat similar. If you don't know how to teach in class, you can't do experiments.

(2) Usually, the experiment is given by the teacher steps, experimental source code and so on, students just carry out the corresponding experimental verification. Causes the student to do the experiment not earnestly, does not actively exercise the experimental ability, to experiment only knows how to do, but does not know why to do so, theory and practice are separated ${ }^{[4]}$.

(3) Some of the teachers who teach embedded system courses are lack of project experience or have never done any related subjects, which leads to the following instructions. Experimental teaching is still taught in the simplest contents such as the oldest GPIO, LED, keyboard and so on. Therefore, students feel that they have not learned anything.

\section{Reform Ideas and Solutions}

Based on the analysis of the disadvantages of the above embedded system and our experience in teaching, training, scientific research and discipline construction in recent years, the basic ideas of the embedded system curriculum reform are given.

\subsection{The curriculum cognition and introduction}

Through displaying the research achievements of the research group for many years, such as linepatrolling robot platform, multi-robot positioning and communication platform, students can have a basic 
understanding of embedded system course. Then the structure, software and hardware of the patrol robot platform are introduced, and the chapters and contents of the embedded system course are corresponded one by one, which makes the students interested in the course. Figure 1 shows the relationship between knowledge points and ARM9 based on line-patrolling robot platform and multi-robot communication and positioning platform.

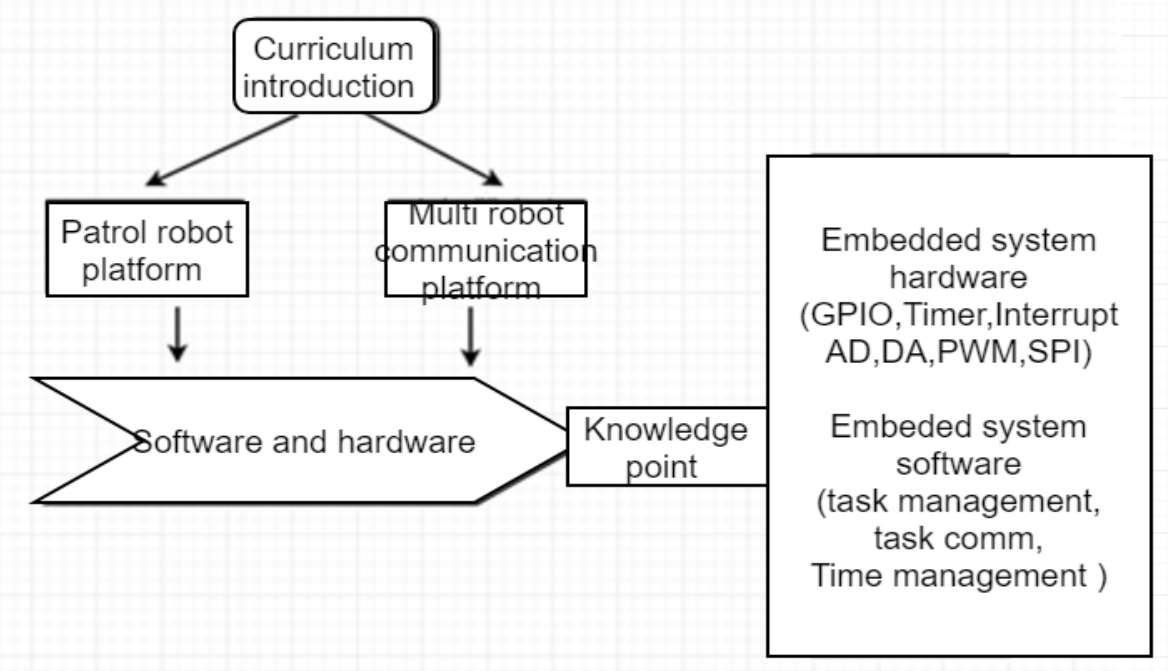

Fig. 1 The correspondence between knowledge points of robot platform and ARM9

\subsection{The project teaching based robot platform.}

According to the characteristics of embedded system course, the course is mainly divided into embedded system hardware foundation and embedded system software foundation (embedded operating system and application part). The details are as follows:

Embedded system hardware basic part describes the basic knowledge of ARM9 SC2440: including timer, interrupt, AD, DA, SPI, PWM, IIC, LCD and other basic knowledge. Each knowledge point applies ADS software integration development platform to design corresponding project subjects for unit consolidation. We introduced the inspection robot platform to carry out the experimental practice of each unit's knowledge points. The application of the timer corresponds to the distance counting measurement of the patrol robot; the interruption is applied to the outside obstacle avoidance of the patrol robot; the AD and DA are applied to the detection of the outside environment information (temperature and humidity) of the patrol robot; and the PWM is applied to the motor control and walking of the patrol robot.

The embedded system software system describes the application of ucOS and Linux operating system. The knowledge points include task management, communication between tasks, time management, operating system transplantation and so on. Similarly, we apply the patrol robot platform to consolidate the knowledge experiment practice of the software part. We choose ucOS operating system under the ADS integrated development environment, and task management is applied to the creation and scheduling of acquisition tasks, image processing tasks, human-machine interface display tasks, motor control tasks and so on. The communication between tasks corresponds to the communication of messages, queues and semaphores among the above tasks of the patrol robot. Time management corresponds to the external interrupt of robot. In particular, the porting part of the operating system we talk about ucOS and Linux porting on the ARM9 platform, mainly through the bootloader programming, file system selection, operating system kernel tailoring and so on. Figure 2 gives a project teaching structure based on the robot platform. 


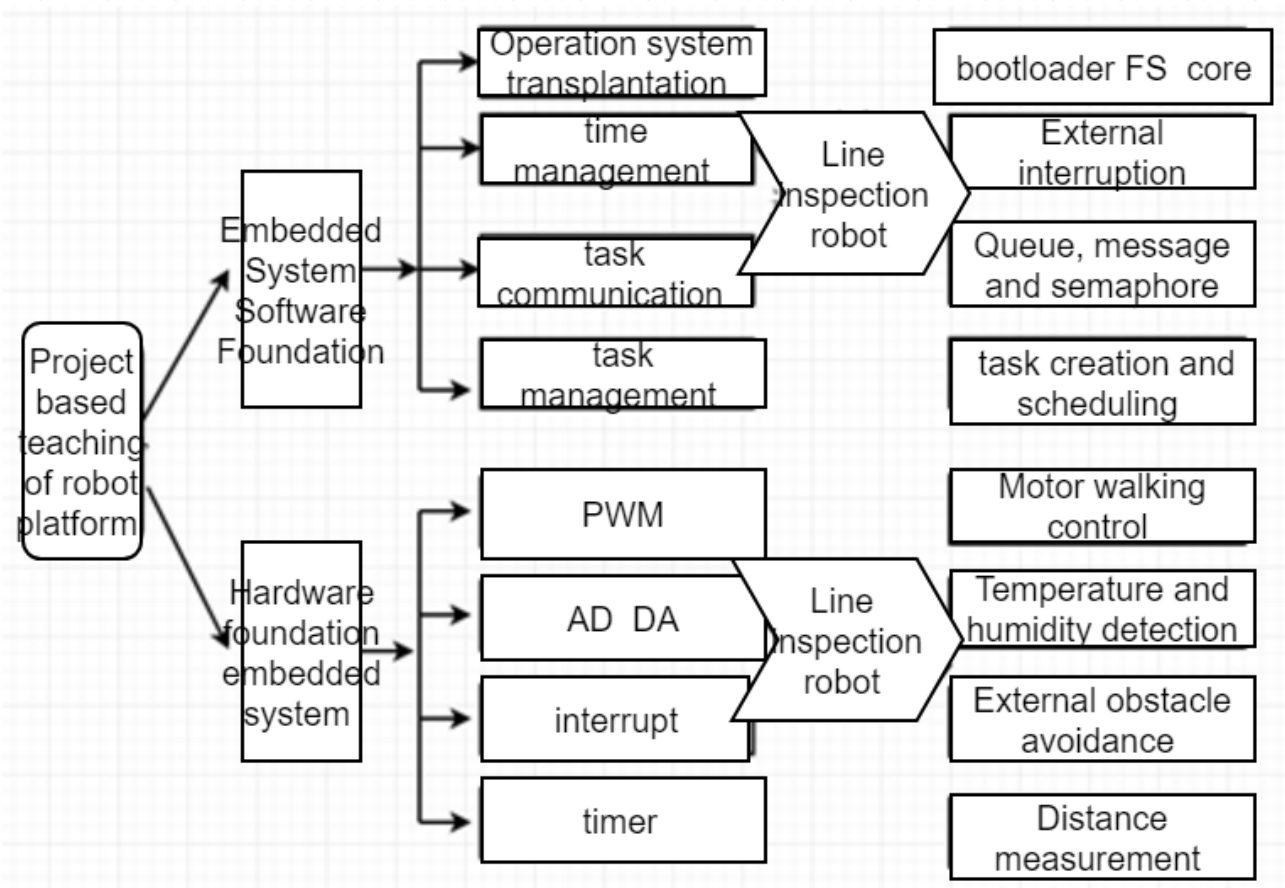

Fig. 2 The project teaching structure based on the robot platform.

\subsection{The improvement of teachers' ability}

In view of the weakness of engineering practice ability of some course teachers, the following reforms and practices have been carried out: firstly, professional teachers are regularly arranged to take part in the product development and production process of enterprises by institutionalizing their post practice in enterprises; secondly, technical personnel of enterprises are employed as part-time teachers and engineering practice education centers are established in enterprises. Let enterprise technicians participate in the training process.

\section{The Curriculum Assessment}

Curriculum assessment is an important part of testing students' learning effect. The results of our course are divided into three parts: (1) 15\% of the students' usual grades, mainly including attendance in class, homework completion, etc. (2) 35\% of the students' robot project scores, mainly including writing project papers, demonstrating project codes and answering relevant code knowledge points, project hardware design principles and methods. Finally, according to the division of labor and internal coordination of the project team members, corresponding scores are given. (3) The final closed-book test results accounted for 50\%, mainly through the examination paper to test students' mastery of basic knowledge points.

\section{Initial Results Achieved}

Through the reform and implementation of the two sessions of students, we have achieved some preliminary results: (1) increased the interest of most students in the embedded system curriculum; (2) students' curriculum performance and hands-on ability has been greatly improved; (3) in employment, 40\% of students choose embedded development, the previous proportion of $10 \%$. The following: (4) The teaching staff has been improved, the original teaching and research room only one person for embedded curriculum teaching, has now fixed four teachers for related teaching, the teaching level of teachers has also made great progress. (5) College began to attach importance to the investment of embedded teaching course funds, related microcontroller, motor, sensor, ARM11 development board, Bochuang robot teaching equipment have been purchased and put into teaching work. 


\section{Acknowledgment}

Foundation Support: The Key Research Projects of Hubei Education Department (D20171503), Teaching Research Projects of Wuhan Engineering University (x2017027)

\section{References}

[1]. Wang Bo, Ji Xiaofu, Jia Haolai. Teaching Reform of "ARM Embedded System" Course [J].Journal of Electrical and Electronic Education, 2015, 37 (3): 18-23.

[2]. Shi Wenjuan. Exploration on the Teaching Reform of Embedded System in Electronic Information Engineering [J]. Forest Area Teaching, 2011 (7): 89-90.

[3]. Zhang Kaiyu, Ludi, Zhou Meilan, et al. Research and Practice of Embedded System Curriculum Reform Based on the Cultivation of Innovative Ability [C]//Heilongjiang Institute of Higher Education. Empirical Study on Improving the Quality of Higher Education in Colleges and Universities (Volume 1). Harbin: Heilongjiang Education Publishing House, 2016:167-170.

[4]. Jiang Wei, Sang Nan. Teaching Reform of Embedded System for Higher Education [J].Computer Education, 2011 (16): 33-36.

[5]. Zeng Ronghua, Luo Yifan, Zhou Hebing, et al. Exploring the construction, opening and management of comprehensive and innovative laboratories [J]. Experimental technology and management, 2010, 27 (9): 2427. 